\title{
THE TREATMENT OF SCIATICA, BRACHIALGIA, AND OCCIPITAL HEADACHE
}

\section{BY RALPH STOCKMAN}

Sciatica-pain in the distribution of the great sciatic nerveoccurs as a symptom in a number of totally different pathological conditions such as pressure on the nerve elements from a pelvic tumour or bony overgrowth in the vertebræ or sacro-iliac and hip joints, in certain spinal cord lesions, or as a result of the action on the nerve fibres of injury or chemical poisons like alcohol and lead, but such cases, although not uncommon, are seldom seen in practice compared with the large number which are due to chronic fibrositis of the muscles and nerves, and it is to the treatment of these that the present remarks apply.

The essential pathology of fibrositis is now well recognised and agreed upon. Under irritation from one source or another the white fibrous tissue of the muscles, nerves, fasciæ and other fibrous structures undergoes inflammatory hyperplasia in small patches, the affected areas become swollen and odematous with sero-fibrinous exudation, the fibroblasts proliferate rapidly, numerous minute new bloodvessels appear, and the whole forms a soft, ill-defined, congested little swelling. In the exudate polymorphonuclear leucocytes are entirely absent, with consequent absence of pus formation. At this soft early stage the small inflamed excrescences may resolve readily under treatment or spontaneously, but more frequently they increase in size from continued proliferation of their connective tissue cells, thereby becoming more fibrous and tougher, the perineurium of the nerve twigs ramifying through them undergoes interstitial inflammation and hypertrophy, and the minute arterioles and veins have their walls greatly thickened. Fibrositic thickenings may also begin insidiously, increasing in size very slowly and gradually and attracting no attention for a long time.

On palpation through the skin the larger ones (the size of a small pea to an almond) can be felt as ill-defined swellings along the edges of muscles or in fascia and subcutaneous tissue, but much of the altered tissue is too minute to be detected in this 
way and can be located only by its tenderness on pressure. The new formations may be scattered in large numbers all over the body or may be confined to one or a few parts.

The original lesion is most commonly the result of bacterial infection, acute rheumatism and influenza being the most frequent although there are many others, while metabolic products and local injury from cold and overstrain are also capable of starting the whole process. Adhesions are formed in this way, usually by local bacterial infection, and in their origin and structure are exactly the same as other fibrositic growths. Whatever the original cause may have been the end-result is the same: the new formations remain as delicate or more or less dense fibrous tissue well supplied with chronically inflamed nerve twigs and thickwalled bloodvessels. This pathological tissue is extremely sensitive to a large number of disturbing influences such as weather changes, cold winds and draughts, unaccustomed muscular exertion or sudden strain, the toxins of slight febrile illnesses and intestinal indigestion, as well as others known and unknown. Exposed to such irritants the thickened bloodvessels become congested, serum exudes and is not readily reabsorbed, the little areas become swollen and tense, pressure is exerted on the hyperæmic ultra-sensitive nerve twigs and there results pain, or in ac lesser degree merely weariness, aching and stiffness. For instance, in a healthy individual unaccustomed exercise (that is, repeated slight traumata from overstrain) irritates the fibrous tissues of the strained muscles to the extent of causing fatigue and aching and stiffness, but, as the nerves are healthy and not hypersensitive pain is not experienced, and, as the bloodvessels are normal congestion and exudation are small in amount and are soon got rid of with or without the help of a hot bath and rubbing. But in a fibrositic subject the effects of unwonted exertion are much more severe and may last for weeks owing to the nerves being hypersensitive and to the pathological bloodvessels failing to deal with the congestion and exudation, and so failing to relieve tension.

By keeping these pathological considerations in mind one is helped to a more precise and individual method of treating cases of sciatica than can be gathered from the textbooks. The advice given in these varies very little from book to book but offers a varied choice, comprising rest in bed, the application of splints, heat, electricity, anodyne liniments, counter-irritants, dry cupping, red rays, ionisation, massage, a great array of palliative and alleged 
curative drugs, diet, climate, local injections of cocaine, ether, chloroform, oxygen, water and saline solution, acupuncture and stretching of the nerve. A heterogeneous mass of procedures like this is, however, very little helpful. Many of them are only useful to allay pain, many are useless, and some of value only in selected cases and properly applied. For successful treatment the remedial measures have to be adapted to the pathological conditions present in each patient, and these vary a good deal.

Judged by my own experience in practice sciatic pain most commonly arises from the presence of rheumatic nodules and thickenings in the buttock, and especially in its upper two-thirds. The pain has its origin in the inflamed hypersensitive twigs from the sciatic nerve which traverse the fibrositic areas, and from them it is reflected down the nerve trunk, which is usually healthy and shows no special tenderness on pressure. On palpating over the skin of the buttock (preferably using a lubricant) numerous little swellings can be felt in connection with the fibrous sheaths of the gluteus medius and maximus lying along and just under the crest of the ilium and lower down. They are very sensitive, and can thus be localised by their tenderness to pressure if they cannot be felt. The basis of treatment is massage of the fibrositic areas in the buttock. As adjuvants, heat applied locally for ten minutes previous to the massage renders it more effective and less painful, and anodyne applications locally and analgesics internally have their own proper application. Any other measures are either unnecessary or useless.

The massage is of a simple kind and a lubricant must be freely used. If the patient cannot afford the services of a professional masseur any intelligent person with a little preliminary instruction and occasional supervision can carry out treatment satisfactorily. The early efforts are directed to getting rid of the effusion and congestion in the inflamed areas, and for this gentle but firm effleurage should be given. It is always painful, and the amount of pressure has to be measured by the patient's capacity to bear pain. The swollen congested tissues bleed easily, and at first there may be a considerable amount of bruising and discoloration of the skin, but this does not interfere with treatment and ceases in two or three weeks. The massage should be given daily for twenty to thirty minutes. At the end of three or four weeks the pain it causes has greatly diminished, and from then on it should be given as hard as the patient can reasonably be expected to 
bear it. By this time the thickenings have become more defined and can be treated individually with hard pressure, making circular movements with the tips of the fingers and knuckles, but these should always be preceded by a few minutes' effleurage to remove fluid and reduce congestion. The hard pressure either disperses the thickenings or breaks up and destroys their bloodvessels and nerves so that they shrink and harden, cease to respond to irritation, and can no longer become congested and swollen and painful. The massage should be continued until it ceases to cause pain, and this may take two or three months from the start. The prognosis is always good unless the nerve trunk is also involved, when further and different treatment is required.

The other common cause of sciatica is fibrositis of the nerve trunk, the usual site being in the buttock and much less frequently the thigh. It is the nerve sheath which is primarily attacked, but the inflammation spreads into the finer connective tissue of the nerve bundles and fibres, causing interstitial neuritis (neurofibrositis), often with excruciating pain and disability in the limb. As the nerve sheath is a firm, dense membrane closely investing the nerve bundles, when it is acutely inflamed the inflammatory exudate does not readily escape and the tension and pressure on the nerve proper may be severe. An acute attack of this kind leaves in its wake permanent fibrositic tissue which? in its turn readily inflames and gives rise to repeated attacks of sciatica. The interstitial neuritis interferes with the axis cylinders, and is thereby responsible for pain and for the numbness, muscular wasting and altered reflexes which sometimes supervene.

The measures to be employed in treating these cases have to be adapted to the local condition. If there is much local reaction in the nerve sheath the indications are to relieve pain, lessen congestion, and promote absorption of the exudate by rest in bed, heat and anodynes locally and analgesics internally. Mild diaphoretics and saline purgation help matters. . If experienced and highly skilled massage is available it should be begun at once or as early as possible, and should consist of the gentlest effleurage. Most writers advise that its use should be avoided in the early stages, but it is to be borne in mind that a septic inflammation of connective tissue differs markedly from a fibrositic one. In the former massage would only do harm as the exudation becomes purulent and the tissues tend to soften and break down, whereas in the latter pus never forms and the exudate tends to organise 
and form more pathological fibrous tissue. The great object of treatment, therefore, is to get rid of the exudate at as early a stage as possible, and for this massage and gentle pressure by bandaging are the best means available so far. The only contra-indication is that it may be too painful, but that depends largely on the masseur. It soon becomes less painful and should be continued as long as it seems to be doing good. But it is difficult to massage away adhesions and inflammatory tissue buried in the soft substance of the buttock, and recourse may have to be had to surgery to clear the nerve sheath and nerve bundles from their fibrositic tissue. It is in such cases that acupuncture, stretching of the nerve, and injection of fluid or oxygen have been found to relieve pain. They act by stretching adhesions and lessening tension, but their action is uncertain, very often only temporary, and they are not curative, as they do not get rid of the inflamed tissue. Careful surgical dissection is in every respect preferable to such haphazard procedure. Surgery, however, cannot reach the fine interstitial neuritis in the nerve trunk, which must be dealt with by the tedious process of deep massage.

Sciatica coming on after gross injury to the nerve is due to adhesions or a cicatrix, and is best treated by an operation for their removal.

BRACHALGIA has the same pathology as sciatica and the same principles of treatment apply, but the actual carrying of them out is easier and the result more speedy. On palpation painful fibrositic spots are found in the fibrous structures connected with the trapezius, deltoid, pectoralis major, supra- and infra-spinatus and other muscles, and from the inflamed nerve twigs traversing these spots pain may be reflected to the trunks of the parent nerves-circumflex, median, ulnar and musculo-spiral. The nerve trunks may be quite healthy, and, if so, treatment need be directed only to the painful muscle or muscles. Very often, however, it is one or other of the nerve trunks in the upper arm which is inflamed and tender, usually over a quite small area. The painful areas in muscle and nerve should be identified and massaged. As a rule no other measures are necessary, as, owing to the small bulk of the muscles and the fact that pressure can be made against bone, the massage is very effective.

HEADACHE.-Dull or acute pain in the occipital, frontal or temporal regions is often due to perineuritis of the nerves supplying these parts. The inflamed area is very small and generally 
confined to the point where the nerve emerges from deep cover: the supraorbital nerve at the supraorbital notch, the temporal as it crosses the temporal fossa, and the great occipital as it runs superficially in the scalp. Massage at these points can readily disperse the new fibrous tissue. Fly blisters are also useful.

But in the occipital region pain and headache frequently arise from fibrositic areas in the neck and shoulder muscles or in the abundant fibrous tissue of the cervical vertebræ, often, therefore, low down in the neck and at a distance from where the pain is felt. The pain is reflected along the larger nerves from small twigs in the inflamed patches. In order that massage may be accurately applied the whole area must be searched, and if the offending places are superficial treatment is satisfactory, but if they are deep in it is often difficult or even impossible to reach them effectively. As these cases are always tedious patients should be instructed to treat themselves for five or ten minutes daily after having had a course from a professional masseur. 